\title{
Avance tendinoso no convencional para lesiones en las zonas I y II de Verdan. Nueva técnica quirúrgica
}

\author{
Alberto L. Garay, Carlos E. Martínez, Juan M. Patiño, Germán Tonetto, Francisco López Bustos \\ Servicio de Miembro Superior, Instituto Dupuytrén, Ciudad Autónoma de Buenos Aires, Argentina
}

\begin{abstract}
RESUMEN
Objetivo: Presentar una nueva técnica quirúrgica y los resultados clínicos comparativos de pacientes con roturas de tenorrafia primaria o lesiones crónicas del flexor profundo en las zonas I y II de Verdan, tratados con avance tendinoso no convencional. Materiales y Métodos: Se incluyó a pacientes >18 años, con antecedente de lesiones en las zonas I y II de Verdan y un seguimiento mínimo de 36 meses. La serie estaba formada por 13 pacientes (edad promedio 29 años) que fueron divididos en dos grupos según el tipo de lesión ( 7 con roturas de tenorrafia primaria y 6 con lesiones crónicas del flexor profundo) y la técnica quirúrgica utilizada (alargamiento en Z más sutura término-terminal y sutura lateral del tendón alargado a un flexor vecino indemne en la zona V, respectivamente). Se empleó la Clasificación de Strickland para la evaluación. Resultados: El seguimiento promedio fue de 51 meses. El intervalo promedio entre la lesión y la cirugía fue de 11.7 semanas. Según la Clasificación de Strickland, 8 pacientes tuvieron resultados excelentes; 4, buenos y uno, pobre. El avance tendinoso promedio fue de 20,5 mm en ambos grupos. Conclusiones: El avance tendinoso no convencional para lesiones en las zonas I y II de Verdan, sea en roturas de tenorrafia primaria o lesiones crónicas del flexor profundo, resultó un tratamiento reproducible y eficaz.
\end{abstract}

Palabras clave: Tendón flexor; alargamiento; zetaplastia.

Nivel de Evidencia: III

Non-conventional tendon advancement for injuries in Verdan zones I and II.

A new surgical technique

\section{ABSTRACT}

Objective: To report a new surgical technique and the comparative clinical outcomes in patients with primary tenorrhaphy rupture or chronic flexor digitorum profundus (FDP) injuries in Verdan zones I and II, treated with non-conventional tendon advancement. Materials and Methods: The study population consisted of 13 patients over 18 years (averaging 29 years) who had had injuries in Verdan zones I and II and at least a 36-month follow-up. Patients were divided into two groups according to injury type (7 cases of primary tenorrhaphy rupture and 6 cases of chronic FDP injuries) and surgical technique (Z-plasty lengthening plus end-to-end suture or lateral suture to an adjacent undamaged FDP tendon at zone-V level, respectively). The results were evaluated according to Strickland's scoring system. Results: The average follow-up was 51 months. Time period between injury and surgery averaged 11.7 weeks (range, 2-24 weeks). Strickland scoring system results: excellent in 8 patients, good in 4 patients, and poor in 1 patient. Study average tendon advancement was $20.5 \mathrm{~mm}$. Conclusions: Non-conventional tendon advancement for primary tenorrhaphy ruptures or chronic FDP injuries in Verdan zones I and II proved to be a reproducible and effective treatment.

Key words: Tendon; flexor; lengthening; Z-plasty.

Level of Evidence: III

\section{INTRODUCCIÓN}

Las lesiones de los tendones flexores de la mano son cuadros prevalentes. Su reparación, avalada por la bibliografía, en forma primaria y en la fase aguda, augura mejores resultados finales. Ante la imposibilidad de realizar una sutura primaria precoz o la rotura de una tenorrafia primaria, el injerto de tendón es el tratamiento de elección.

Recibido el 30-7-2020. Aceptado luego de la evaluación el 2-8-2020 • Dr. ALBERTO L. GARAY • albertogaray146@ hotmail.com ID https://orcid.org/0000-0001-6371-0884 Cómo citar este artículo: Garay AL, Martínez CE, Patiño JM, Tonetto G, López Bustos F. Avance tendinoso no convencional para lesiones en las zonas I y II de Verdan. Nueva técnica quirúrgica. Rev Asoc Argent Ortop Traumatol 2020;85(Supl.):S12-S18. https://doi.org/10.15417/issn. 1852-7434.2020.85.4S.1173 
Las tasas de adherencias y roturas (principales complicaciones comunicadas) en la sutura primaria o secundaria, así como la comorbilidad inherente al uso de injertos tendinosos, ya sea del antebrazo o del miembro inferior, son factores ampliamente conocidos, respetando la regla lógica de que, a mayor número de cirugías, más limitación residual. ${ }^{1-3}$

Con el fin de buscar una alternativa a los tratamientos convencionales, informes de las décadas de 1950 y 1980 describieron resultados alentadores de los alargamientos del flexor largo del pulgar en la zona $\mathrm{V}$ de Verdan en casos de rotura. ${ }^{4,5}$

Por su parte, Le Viet realizó zetaplastias de flexores profundos en la retracción del muñón proximal para lograr una sutura primaria. ${ }^{6}$

Más recientemente, este procedimiento de alargamiento tendinoso no convencional se utilizó para tratar lesiones de tendones flexores de los dedos trifalángicos por arrancamiento (jersey finger). ${ }^{7}$

El objetivo de este estudio es comunicar una nueva técnica quirúrgica y los resultados clínicos comparativos de pacientes con roturas de tenorrafia primaria o lesiones crónicas del flexor profundo en las zonas I y II de Verdan, tratados con avance tendinoso no convencional.

\section{MATERIALES Y MÉTODOS}

Se llevó a cabo un estudio retrospectivo que evaluó a pacientes tratados entre diciembre de 2012 y marzo de 2018. Los criterios de inclusión fueron: edad $>18$ años, antecedente de rotura de tenorrafia primaria o de lesiones crónicas del flexor profundo en las zonas I y II de Verdan, en dedos trifalángicos, con lesiones asociadas de nervios digitales o sin ellas y un seguimiento mínimo de 36 meses. Se excluyó a los que no cumplían estos criterios, tenían defectos de partes blandas, fracturas o procesos infecciosos.

La serie incluyó a 13 pacientes (9 hombres y 4 mujeres), con una edad promedio de 29 años (rango 20-49). El $85 \%$ de los casos (11 pacientes) tenía compromiso de la mano dominante. El dedo más afectado era el meñique (6 casos), seguido del índice (3 casos) y los dedos medio ( 2 casos) y anular ( 2 casos). En cuatro casos (30\%), se realizó una neurorrafia de colaterales lesionados. Este procedimiento retrasó el período de rehabilitación.

Las cirugías estuvieron a cargo de dos especialistas en cirugía de mano, se efectuaron con anestesia general o bloqueo plexual, en un solo tiempo quirúrgico, ya que no se registraron adherencias de muñones ni fue necesaria la reconstrucción de poleas.

Se dividió a la muestra en dos grupos según el tipo de lesión y la técnica quirúrgica utilizada. El grupo A estaba formado por pacientes con roturas de tenorrafia primaria (7 casos). La técnica quirúrgica consistió en una zetaplastia en la zona $\mathrm{V}$, en el cabo proximal del tendón comprometido, prolongando, de esta forma, su longitud. Así se logra alcanzar, en las zonas I o II de Verdan, el cabo distal, para hacer allí la correspondiente sutura término-terminal (Figura 1). El avance del tendón se realiza a través de las poleas correspondientes al tendón que se repara. El cabo proximal puede suturarse al distal o insertarse en la falange distal mediante la técnica de pull-out.

El grupo B incluyó a pacientes con lesiones crónicas no tratadas del flexor profundo (6 casos). Si el acortamiento del cabo proximal es considerable y, por lo tanto, no se puede realizar la sutura término-terminal inicialmente, proponemos la zetaplastia sobre el cabo proximal, avance y sutura término-terminal a distal y suturar al tendón del flexor profundo vecino indemne en la zona $\mathrm{V}$ con sutura continua (a proximal) (Figura 2). La razón de realizar las suturas en la zona $\mathrm{V}$ es la menor tasa de adherencias descrita.

\section{Cuidados posoperatorios}

Después de la cirugía, a ambos grupos, se les indicó inmovilización con yeso por encima del codo para proteger la sutura, durante cuatro semanas. Los ejercicios de rehabilitación comenzaron después de retirar el yeso y se aplicó una carga progresiva a la mano, a las ocho semanas de la cirugía.

Se utilizó la Clasificación de Strickland ${ }^{8}$ para evaluar con goniometría, en forma objetiva, los resultados, registrando el rango de movilidad activa total, definido como la suma de la flexión activa de las articulaciones metacarpofalángica, interfalángica proximal y distal, menos el déficit de extensión de dichas articulaciones. Los resultados se consideraron excelentes si $>150^{\circ}$, buenos de $125^{\circ}$ a $149^{\circ}$, regulares de $90^{\circ}$ a $124^{\circ}$ y malos $<90^{\circ}$.

Para el análisis estadístico se empleó el programa IBM SPSS Statistics 19.0 sobre el alargamiento obtenido en cada grupo. Este análisis se desarrolló con una prueba mediana no paramétrica. Se consideró como significativo un valor $\mathrm{p}<0,05$. 

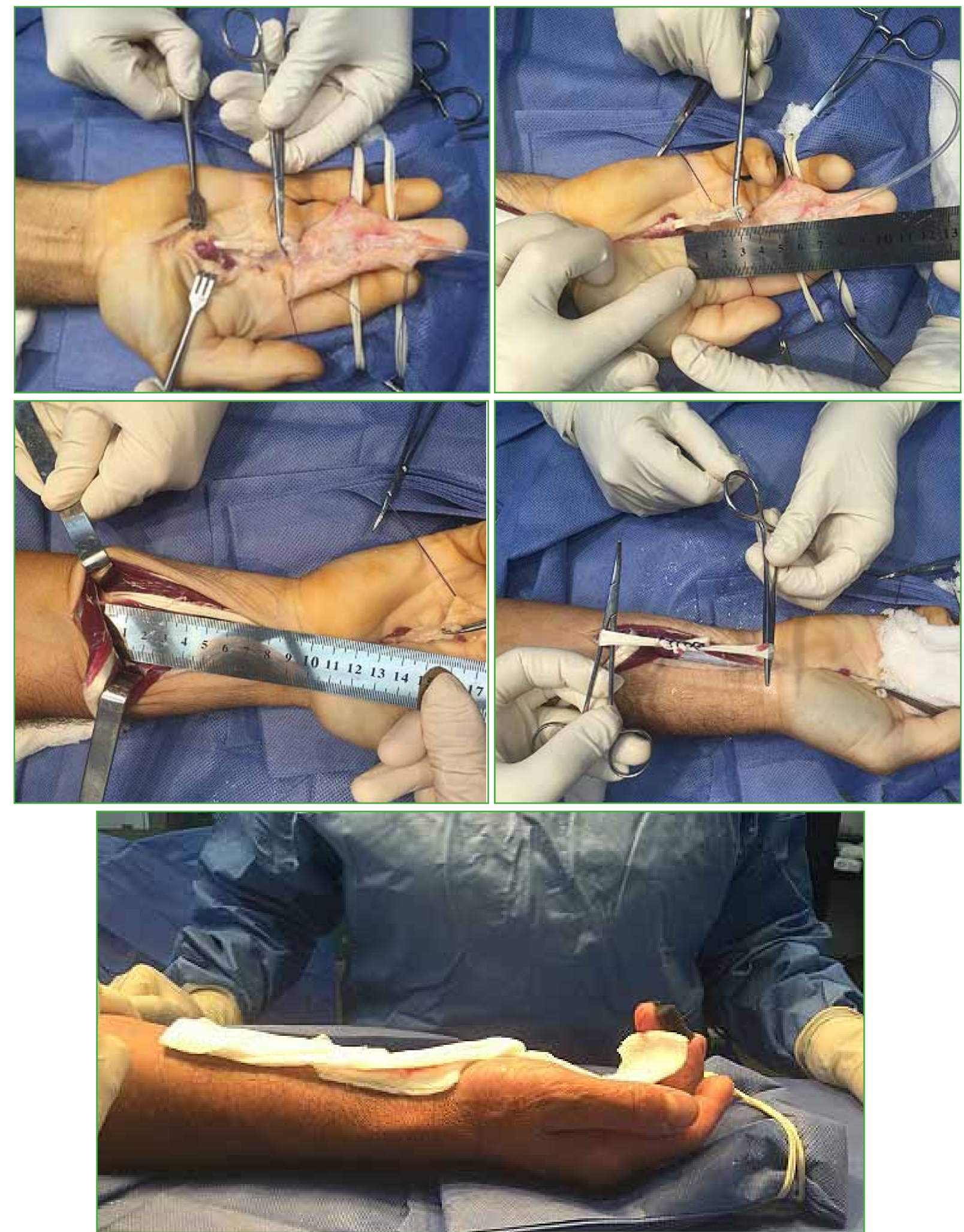

Figura 1. Secuencia intraoperatoria de la técnica no convencional de alargamiento tendinoso, utilizada en roturas de tenorrafias primarias. 


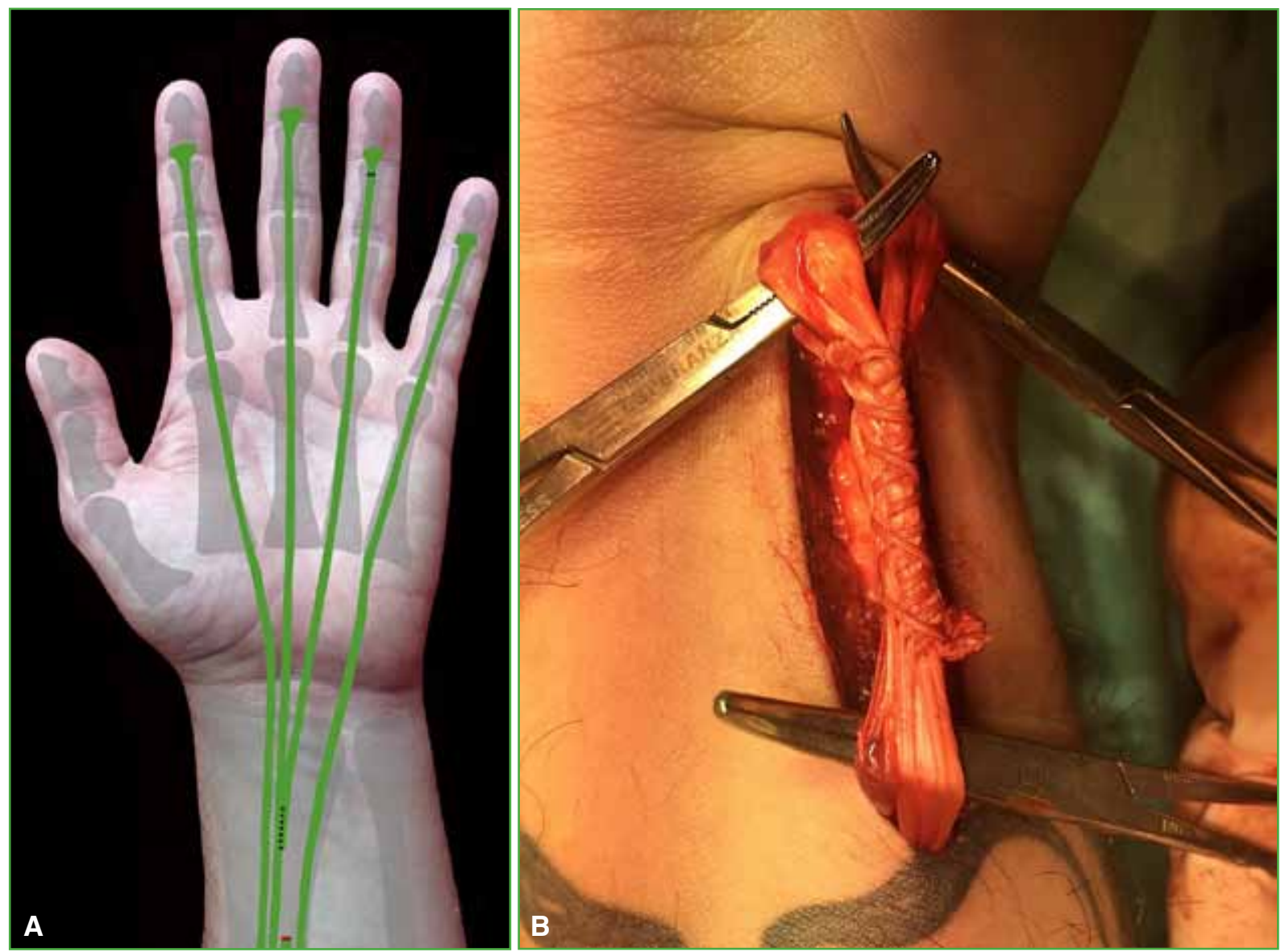

Figura 2. Esquema de tenodesis del flexor retraído al tendón contiguo sano (A) e imagen intraoperatoria de la sutura del alargamiento (B), utilizada en lesiones crónicas del flexor profundo.

\section{RESULTADOS}

El seguimiento promedio fue de 51 meses (rango 41-62). El intervalo promedio entre la rotura o lesión y la cirugía fue de 11.7 semanas (rango 2-24). Según la Clasificación de Strickland, en el grupo A (7 pacientes), cinco tenían un rango de movilidad activa total excelente; uno, bueno y otro, regular. En tanto, en el grupo B (6 pacientes), tres obtuvieron resultados excelentes y tres resultados buenos. Los datos demográficos, el promedio de seguimiento y la Clasificación de Strickland de ambos grupos se resumen en las Tablas 1 y 2.

El avance promedio logrado para los tendones tratados fue de 20,5 mm (rango 12-32) en los dos grupos. El grupo A registró un avance promedio de $18 \mathrm{~mm}$, y el grupo B, de 23,5 mm. Estas diferencias fueron estadísticamente significativas $(\mathrm{p}=0,029)$. Los datos se resumen en la Tabla 3. No se registraron complicaciones ni nuevas operaciones en ninguno de los grupos.

\section{DISCUSIÓN}

La reconstrucción del tendón flexor profundo está indicada cuando fracasan las reparaciones primarias, si el diagnóstico de retracción del cabo proximal es tardío y para lesiones complejas con defecto tegumentario. En esta serie de pacientes, se comparó el resultado funcional de dos técnicas de avance tendinoso no convencional para lesiones en las zonas I y II de Verdan, en dedos trifalángicos; en ambos casos, se logró un buen rango de movilidad activo total al final del seguimiento, al margen de las distintas situaciones.

El pronóstico en las reconstrucciones está determinado por la demora en el tratamiento, la tasa de retracción del tendón, su estado de vascularización, el entorno de los tejidos blandos, la indemnidad del tendón flexor superficial y la experiencia del cirujano. 
Tabla 1. Serie de pacientes tratados por roturas de tenorrafia primaria (grupo A)

\begin{tabular}{|c|c|c|c|c|c|c|c|c|c|}
\hline Caso & Sexo & $\begin{array}{l}\text { Edad } \\
\text { (años) }\end{array}$ & Etiología & $\begin{array}{l}\text { Dedo, lado, } \\
\text { dominancia }\end{array}$ & Técnica & $\begin{array}{l}\text { Intervalo } \\
\text { lesión- } \\
\text { cirugía } \\
\text { (semanas) }\end{array}$ & $\begin{array}{l}\text { Seguimiento } \\
\text { (meses) }\end{array}$ & $\begin{array}{c}\text { Avance } \\
\text { (mm) }\end{array}$ & $\begin{array}{l}\text { Clasifica- } \\
\text { ción } \\
\text { de Stric- } \\
\text { kland }\end{array}$ \\
\hline 1 & M & 25 & $\begin{array}{l}\text { Falla en } \\
\text { la sutura } \\
\text { primaria }\end{array}$ & $\begin{array}{l}\text { 5. }{ }^{\circ} \text {, derecho, } \\
\text { dominante }\end{array}$ & Zetaplastia & 16 & 59 & 20 & Excelente \\
\hline 2 & M & 23 & $\begin{array}{l}\text { Falla en } \\
\text { la sutura } \\
\text { primaria }\end{array}$ & $\begin{array}{c}\text { 5. }{ }^{\circ}, \mathrm{iz}- \\
\text { quierdo, } \\
\text { dominante }\end{array}$ & Zetaplastia & 12 & 59 & 12 & Excelente \\
\hline 3 & M & 25 & $\begin{array}{l}\text { Falla en } \\
\text { la sutura } \\
\text { primaria }\end{array}$ & $\begin{array}{c}\text { 2. }{ }^{\circ} \text {, derecho, } \\
\text { dominante }\end{array}$ & Zetaplastia & 6 & 51 & 15 & Regular \\
\hline 4 & $\mathrm{~F}$ & 49 & $\begin{array}{l}\text { Falla en } \\
\text { la sutura } \\
\text { primaria }\end{array}$ & $\begin{array}{l}\text { 2. }{ }^{\circ} \text {, derecho, } \\
\text { dominante }\end{array}$ & Zetaplastia & 2 & 57 & 15 & Excelente \\
\hline 5 & $\mathrm{~F}$ & 34 & $\begin{array}{l}\text { Falla en } \\
\text { la sutura } \\
\text { primaria }\end{array}$ & $\begin{array}{l}\text { 5. }^{\circ} \text {, derecho, } \\
\text { dominante }\end{array}$ & Zetaplastia & 24 & 50 & 20 & Bueno \\
\hline 6 & M & 35 & $\begin{array}{l}\text { Falla en } \\
\text { la sutura } \\
\text { primaria }\end{array}$ & $\begin{array}{c}4^{\circ} \text {, izquier- } \\
\text { do }\end{array}$ & Zetaplastia & 5 & 54 & 30 & Excelente \\
\hline 7 & M & 36 & $\begin{array}{l}\text { Falla en } \\
\text { la sutura } \\
\text { primaria }\end{array}$ & $\begin{array}{l}\text { 5. }, \text { derecho, } \\
\text { dominante }\end{array}$ & Zetaplastia & 20 & 49 & 22 & Excelente \\
\hline
\end{tabular}

$\mathrm{M}=$ masculino, $\mathrm{F}=$ femenino.

Tabla 2. Serie de pacientes tratados por lesiones crónicas del tendón flexor profundo (grupo B)

\begin{tabular}{|c|c|c|c|c|c|c|c|c|c|}
\hline Caso & Sexo & $\begin{array}{l}\text { Edad } \\
\text { (años) }\end{array}$ & Etiología & $\begin{array}{l}\text { Dedo, lado, } \\
\text { dominancia }\end{array}$ & Técnica & $\begin{array}{l}\text { Intervalo } \\
\text { lesión- } \\
\text { cirugía } \\
\text { (semanas) }\end{array}$ & $\begin{array}{c}\text { Seguimien- } \\
\text { to } \\
\text { (meses) }\end{array}$ & $\begin{array}{l}\text { Avance } \\
\text { (mm) }\end{array}$ & $\begin{array}{l}\text { Clasifica- } \\
\text { ción } \\
\text { de } \\
\text { Strickland }\end{array}$ \\
\hline 1 & $\mathrm{~F}$ & 24 & $\begin{array}{l}\text { Lesión } \\
\text { crónica }\end{array}$ & $\begin{array}{l}\text { 3. }{ }^{\circ} \text {, derecho, } \\
\text { dominante }\end{array}$ & $\begin{array}{c}\text { Unión } \\
\text { intertendinosa }\left(4^{\circ}\right)\end{array}$ & 16 & 62 & 32 & Excelente \\
\hline 2 & M & 22 & $\begin{array}{l}\text { Lesión } \\
\text { crónica }\end{array}$ & $\begin{array}{l}\text { 5. }{ }^{\circ} \text {, derecho, } \\
\text { dominante }\end{array}$ & $\begin{array}{c}\text { Unión } \\
\text { intertendinosa }\left(4^{\circ}\right)\end{array}$ & 6 & 41 & 22 & Bueno \\
\hline 3 & $\mathrm{~F}$ & 27 & $\begin{array}{l}\text { Lesión } \\
\text { crónica }\end{array}$ & 3. ${ }^{\circ}$, derecho & $\begin{array}{c}\text { Unión } \\
\text { intertendinosa }\left(4^{\circ}\right)\end{array}$ & 6 & 52 & 18 & Bueno \\
\hline 4 & M & 26 & $\begin{array}{l}\text { Lesión } \\
\text { crónica }\end{array}$ & $\begin{array}{l}\text { 5. }{ }^{\circ} \text {, derecho, } \\
\text { dominante }\end{array}$ & $\begin{array}{c}\text { Unión } \\
\text { intertendinosa }\left(4^{\circ}\right)\end{array}$ & 24 & 49 & 24 & Excelente \\
\hline 5 & M & 36 & $\begin{array}{l}\text { Lesión } \\
\text { crónica }\end{array}$ & $\begin{array}{l}\text { 5. }{ }^{\circ} \text {, derecho, } \\
\text { dominante }\end{array}$ & $\begin{array}{c}\text { Unión } \\
\text { intertendinosa }\left(4^{\circ}\right)\end{array}$ & 20 & 49 & 22 & Excelente \\
\hline 6 & M & 20 & $\begin{array}{l}\text { Lesión } \\
\text { crónica }\end{array}$ & $\begin{array}{l}\text { 4. }{ }^{\circ} \text {, derecho, } \\
\text { dominante }\end{array}$ & $\begin{array}{c}\text { Unión } \\
\text { intertendinosa }\left(3^{\circ}\right)\end{array}$ & 4 & 50 & 25 & Bueno \\
\hline
\end{tabular}


Tabla 3. Promedio de avance tendinoso obtenido según el grupo

\begin{tabular}{|c|c|c|c|c|}
\hline & Paciente & Grupo A $(\mathbf{m m})$ & Paciente & 22 \\
\hline & 1 & 20 & 1 & 30 \\
\hline & 2 & 12 & 2 & 18 \\
\hline & 3 & 15 & 3 & 24 \\
\hline & 4 & 32 & 4 & 22 \\
\hline
\end{tabular}

Las opciones quirúrgicas clásicas incluyen avance del tendón, injertos en uno o dos procedimientos, transferencias de tendones y prótesis silásticas. Otras cirugías de rescate muy utilizadas son artrodesis, capsulodesis y tenodesis.

Según la bibliografía, los injertos en todas sus variantes son los más elegidos. Sin embargo, no hay consenso sobre qué tendón sería el mejor injerto. Para su elección es preciso considerar el diámetro, la longitud y la resistencia. Los más utilizados son el flexor palmaris brevis, el extensor digiti quinti, el extensor digiti indicis y los extensores de dedos de los pies. ${ }^{9-11}$ También se han propuesto los aloinjertos. ${ }^{12}$

En una serie de 71 casos, Guimberteau y cols. proponen emplear injertos vascularizados de tendones flexores y comunican resultados alentadores, aunque con poco tiempo de seguimiento. ${ }^{13}$

Asimismo, se ha descrito la implementación de implantes sintéticos como herramienta para tratar lesiones de flexores, siempre que sean bien tolerados..$^{13-16}$

Sita-Alb y Durand propusieron la reconstrucción de tendones flexores en las zonas I y II mediante transferencia de la mitad del tendón flexor superficial adyacente al tendón flexor profundo lesionado. ${ }^{17}$

Hay publicaciones sobre el alargamiento del tendón en el antebrazo. ${ }^{6}$ Chanel y cols. ${ }^{7}$ evaluaron el avance del tendón flexor profundo al realizar una zetaplastia como tratamiento para la lesión por arrancamiento (jersey finger). Este avance del tendón fue limitado debido a la presencia del músculo lumbrical y la adherencia sinovial. No se informaron restricciones en el índice. Aunque esta técnica se propuso para este último cuadro, no dejamos de considerar que la zetaplastia podría debilitar el tendón flexor y aumentar la tasa de adherencia.

Cualquiera de estos procedimientos descritos requiere de una técnica muy cuidadosa y amplia experiencia en la reparación de tendones flexores.

La fortaleza de la técnica propuesta reside principalmente en su menor complejidad en comparación con un injerto tendinoso, ya que prescinde de la toma de dicho injerto y, de esta forma, disminuye considerablemente la morbilidad y el tiempo de cirugía. Otra virtud de esta técnica es su versatilidad, porque se puede realizar en uno o dos pasos (determinados por el cirujano siempre que haya adherencias o el requisito de reconstrucción de poleas). Estos beneficios pueden ser mayores si más de dos dedos requieren reparación. Amparados en la preservación de la irrigación del tendón y la similitud de los diámetros anatómicos de los muñones, confirmamos que el deslizamiento del tendón a través de las poleas no se ve comprometido.

Consideramos como limitantes de este estudio su diseño retrospectivo, la serie acotada de pacientes, junto con la heterogeneidad de las lesiones (roturas de tenorrafia primaria y lesiones no tratadas del flexor profundo) y posibles sesgos en la interpretación de los resultados.

\section{CONCLUSIONES}

La técnica de avance tendinoso no convencional para lesiones en las zonas I y II de Verdan, en dedos trifalángicos de la mano, de pacientes con roturas de tenorrafia primaria o lesiones crónicas del flexor profundo, resultó un tratamiento reproducible y eficaz. 
Conflicto de intereses: Los autores no declaran conflictos de intereses.

ORCID de C. E. Martínez: https://orcid.org/0000-0002-6031-0532 ORCID de J. M. Patiño: https://orcid.org/0000-0002-9036-0442
ORCID de G. Tonetto: https://orcid.org/0000-0002-1740-3792 ORCID de F. López Bustos: https://orcid.org/0000-0002-2504-2026

\section{BIBLIOGRAFÍA}

1. Finsen V. Two-stage grafting of digital flexor tendons: a review of 43 patients after 3 to 15 years. Scand J Plast Reconstr Surg Hand Surg 2003;37:159-62. https://doi.org/10.1080/03844310310007773

2. LaSalle WB, Strickland JW. An evaluation of the two-stage flexor tendon reconstruction technique. J Hand Surg Am 1983;8:263-7. https://doi.org/10.1016/s0363-5023(83)80155-5

3. Boyes JH, Stark HH. Flexor tendon grafts in the fingers and thumb. A study of factors influencing results in 1000 cases. J Bone Joint Surg Am 1971;53:1332-42. PMID: 5114696

4. Rouhier G. Restoration of the long flexor tendon of the thumb without sacrificing the primary tendon. J Chir 1950;66:537-42. PMID: 15436545

5. Vigliani F, Martinelli B. Repair of rupture of flexor pollicis longus by “Z” lengthening at the wrist. Ital J Orthop Traumatol 1981;7:171-9. PMID: 7347329

6. Le Viet D. Flexor tendon lengthening by tenotomy at the musculotendinous junction. Ann Plast Surg 1986;17:23946. https://doi.org/10.1097/00000637-198609000-00010

7. Chanel L, Grolleau J, Lauwers F, André A. Z-plasty lengthening of the flexor digitorum profundus at the wrist (zone 5) for the treatment of jersey finger: Anatomical study and evaluation of advancement obtained. J Plast Reconstr Aesthet Surg 2014;67:e189-e194. https://doi.org/10.1016/j.bjps.2014.04.025

8. Strickland JW. Results of flexor tendon surgery in zone II. Hand Clin 1985;1:167-79. PMID: 4093459

9. Wehbé MA. Tendon graft anatomy and harvesting. Orthop Rev 1994;23:253-6. PMID: 8022646

10. Carlson G, Botte M, Josephs M, Newton P, Davis J, Woo S. Morphologic and biomechanical comparison of tendons used as free grafts. J Hand Surg Am 1993;18:76-82. https://doi.org/10.1016/0363-5023(93)90249-3

11. Paneva-Holevich E. Two-stage reconstruction of the flexor tendons. Int Orthop 1982;6(2):133-8. https://doi.org/10.1007/BF00268657

12. Xie RG, Tang JB. Allograft tendon for second-stage tendon reconstruction. Hand Clin 2012;28:503-9. https://doi.org/10.1016/j.hcl.2012.08.011

13. Guimberteau J, Bakhach J, Panconi B, Rouzaud S. A fresh look at vascularized flexor tendon transfers: concept, technical aspects and results. J Plast Reconstr Aesthet Surg 2007;60(7):793-810. https://doi.org/10.1016/j.bjps.2007.02.021

14. Hunter JM, Singer DI, Jaeger SH, Mackin EJ. Active tendon implants in flexor tendon reconstruction. J Hand Surg 1988;13(6):849-59. https://doi.org/10.1016/0363-5023(88)90259-6

15. Hunter JM, Jaeger SH. Tendon implants: primary and secondary usage. Orthop Clin North Am 1977;8(2):473-89. PMID: 142944

16. Honner R, Meares A. A review of 100 flexor tendon reconstructions with prosthesis. Hand 1977;9(3):226-31. https://doi.org/10.1016/s0072-968x(77)80106-x

17. Sita-Alb L, Durand S. Tendon reconstruction with adjacent finger hand tendon. Hand Clin 2013;29(2):243-50. https://doi.org/10.1016/j.hcl.2013.02.006 